\title{
ILCEA
}

Revue de l'Institut des langues et cultures

d'Europe, Amérique, Afrique, Asie et Australie

29 | 2017

Les femmes en Russie : parcours, mythes et représentations

\section{Au-delà des générations. Pistes de réflexion sur la représentation du personnage de la grand-mère dans la littérature russe contemporaine}

СКВОЗЬ ПОКОЛЕНИЯ. РАЗМЫШЛЕНИЯ ОБ ОБРАЗЕ БАБУШКИ В СОВРЕМЕННОЙ

РУССКОЙ ЛИТЕРАТУРЕ

\section{Giulia Gigante}

\section{OpenEdition}

Journals

Édition électronique

URL : http://journals.openedition.org/ilcea/4250

DOI : 10.4000/ilcea.4250

ISSN : 2101-0609

Éditeur

UGA Éditions/Université Grenoble Alpes

Édition imprimée

ISBN : 978-2-37747-007-5

ISSN : 1639-6073

\section{Référence électronique}

Giulia Gigante, «Au-delà des générations. Pistes de réflexion sur la représentation du personnage de la grand-mère dans la littérature russe contemporaine », ILCEA [En ligne], 29 | 2017, mis en ligne le 30 juin 2017, consulté le 01 mai 2019. URL : http://journals.openedition.org/ilcea/4250 ; DOI : 10.4000/ ilcea. 4250

Ce document a été généré automatiquement le 1 mai 2019.

(C) ILCEA 


\section{Au-delà des générations. Pistes de réflexion sur la représentation $\mathrm{du}$ personnage de la grand-mère dans la littérature russe contemporaine}

СКВОЗЬ ПОКОЛЕНИЯ. РАЗМЫШЛЕНИЯ ОБ ОБРАЗЕ БАБУШКИ В СОВРЕМЕННОЙ

РУССКОЙ ЛИТЕРАТУРЕ

\section{Giulia Gigante}

« Ma grand-mère avait l'habitude de dialoguer avec les objets, parfois en dialecte, parfois dans des langues différentes et totalement inventées parce que, comme elle m'expliquait avec le plus grand sérieux, une chaise ne parle pas comme un piano ou comme une casserole. " Andrea CAMILLERI

Parmi les nombreux personnages qui peuplent la littérature russe contemporaine, l'image de la babouchka se distingue par son importance. Cela est dû non seulement à la personnalité des grand-mères et aux valeurs qu'elles relayent, mais aussi au rôle qu'elles ont joué, et parfois jouent encore, dans la société russe en vertu de circonstances historiques particulières et de l'organisation de la vie sociale, surtout à l'époque soviétique ${ }^{1}$. Pour ces raisons il est intéressant d'analyser les fonctions littéraires que l'on peut associer à ce personnage. En recensant l'entretien d'Irina Denezhkina avec sa grandmère (Denežkina, 2006) - que l'écrivaine considère comme l'un des « héros de notre temps»-, le critique littéraire Alexandre Ageev se demande, avec un soupçon d'exagération: "Seul le diable le sait, et si la culture russe reposait justement sur les grand-mères ${ }^{2}$ ?» Même sans aller jusque-là, il faut reconnaître que l'auteur-démiurge accorde souvent à ce personnage un rôle de premier plan dans l'univers poétique qu'il crée. 


\title{
Une charnière entre le présent et le passé
}

\author{
«Charme profond, magique, dont nous grise \\ Dans le présent le passé restauré !» \\ Charles BAUDELAIRE \\ « Le passé est une terre étrangère. » \\ Gianrico CARofiglio
}

2 L'un des aspects les plus intéressants de la figure de la babouchka qui ressort de l'examen des œuvres de quelques auteurs de la période de la seconde moitié du xxe siècle à nos jours, c'est sa fonction de charnière entre des temps et des lieux différents. Les écrivains ont tendance à confier aux grand-mères qu'ils représentent dans leurs pages la mission difficile de forger un lien avec le passé et d'assurer la continuité sur le plan historique et culturel au sens large. C'est un procédé auquel Ludmila Oulitskaïa recourt souvent. À travers des personnages comme Elizaveta Ivanovna dans Sincèrement vôtre, Chourik, et Anna Aleksandrovna dans Le chapiteau vert, elle propose une liaison avec la culture du début de siècle en évoquant, par leurs gestes et leurs paroles, un patrimoine d'humanité, d'idéaux et de simple gestion du quotidien à l'opposé de la doctrine officielle et en suggérant une autre façon de vivre et surtout une possibilité de regarder et interpréter la réalité autrement.

3 Le pont vers le passé est jeté dans Sincèrement vôtre, Chourik par la narration d'histoires de famille, étayée par des photographies et des coupures de journaux. L. Oulitskaïa utilise la métaphore du fil de la pelote pour décrire comment les récits de la grand-mère se dénouent : "Avec les années ses merveilleuses histoires se figèrent, s'engourdirent et elles paraissaient demeurer comme des pelotes invisibles dans le tiroir de son secrétaire à côté des photographies ${ }^{3}$.»

4 Avec son chignon démodé et la dentelle autour de son cou, Elizaveta Ivanovna ramène à la surface non seulement des gens et des faits d'antan, mais aussi des coutumes et des valeurs devenues désuètes comme, par exemple, la tolérance. Par sa méthode didactique, qui fait d'elle une enseignante sans pareil, la vieille dame transmet non seulement sa connaissance de la langue française à son petit-fils et à d'innombrables élèves, pour lesquels cet idiome devient une deuxième langue maternelle, mais aussi sa vénération pour les poètes français.

D'une manière analogue, c'est la grand-mère de Sanja, l'un des protagonistes du Chapiteau vert, qui apprend à son petit-fils et à ses amis l'importance de la littérature et de la culture en général et initie le garçon à la musique, perçue comme nourriture de l'âme et air vital. La musique représente l'accès à un monde supérieur, élevé, qui s'oppose à l'insupportable vulgarité du quotidien. Grâce à Anna Aleksandrovna, on respire dans cette maison une atmosphère tout à fait différente de celle des années soviétiques : on y rencontre des gens hors du commun ; les conversations, souvent sur des thèmes élevés, se déroulent dans le respect réciproque; on y trouve une riche bibliothèque avec des textes précieux (et aussi des livres interdits comme l'Évangile) ; on sert le thé et on prépare les biscuits à l'ancienne. C'est peut-être pour cela que Mikha, dès qu'il connaît la grand-mère de son ami Sanja, tombe amoureux d'elle.

Médée, la protagoniste de Médée et ses enfants, peut être considérée elle aussi comme une grand-mère sui generis pour le rôle de matriarche qu'elle joue alors même qu'elle n'a pas 
d'enfants mais une nuée de neveux et nièces ; non seulement elle subvient à la nourriture matérielle et spirituelle des nombreux membres de sa famille - ce qui est un caractère distinctif de la figure de la babouchka selon Irina Savkina ${ }^{4}$-, mais préserve et alimente des traditions et des valeurs du temps passé. Celui-ci est pour l'hérö̈ne une mine inépuisable d'histoires à conter et une ressource où puiser la force pour résister aux intempéries de la vie, le courage d'accomplir des choix difficiles, tant sur le plan personnel que social (par exemple, dans le cas de l'héroïne, la solidarité qu'elle manifeste pour les Tatars de Crimée). L'importance du passé pour Médée est symboliquement représentée par une malle en cuir où se cachent, en plus des objets utiles et inutiles accumulés à différentes époques, des reliques de familles, des photographies anciennes, des mèches de cheveux et, surtout, des piles de vieilles lettres. C'est une véritable archive de famille où l'héroïne peut se refugier dans les moments de crise pour y chercher le réconfort et l'oubli : "C'étaient de véritables archives familiales, et comme toutes les vraies archives, elles recelaient des secrets attendant leur heure pour être révélés ${ }^{5}$.»

7 Dans son roman Le temps des femmes, Elena Tchijova nous propose des voix de femmes qui s'alternent, et qui expriment des points de vue différents. Ce sont trois grand-mères d'adoption pour lesquelles l'auteur a choisi - et ce n'est pas un hasard - des prénoms démodés, un peu désuets : la grincheuse Evdokia, la joyeuse Glikeria et la douce Ariadna. Dans l'espace suffocant à huis clos d'une kommunalka (appartement communautaire) du Léningrad de l'après-guerre, les trois femmes prennent en charge une petite fille apparemment muette. Comme des Parques, elles coupent et filent la destinée de cette petite fille et, puisant dans leurs souvenirs et dans des ressources qu'elles ignoraient avoir, elles lui inventent une vie beaucoup plus riche sur le plan spirituel que celle qu'elle aurait eue sans leurs soins et, en même temps, donnent un sens à leur propre existence. L'histoire de la Russie soviétique se déroule devant le lecteur dans tout son caractère dramatique, filtrée à travers les vicissitudes privées des trois femmes et de leurs proches. Les années terribles de la guerre, les affres de la faim, le froid et les déportations ne sont jamais racontés directement, mais transparaissent dans les discours murmurés dans la cuisine, entre une soupe préparée avec des restes et une broderie, un conte de fée ou une comptine narrés à la petite et une prière chuchotée. Elles sont vécues à travers les heurs et malheurs des personnages. Ces conversations évoquent les histoires que E. Tchijova avait à son tour entendues dans son enfance, à l'heure du thé dans la cuisine, de sa mère et sa grand-mère qui avaient perdu presque tous leurs proches pendant le siège de Léningrad.

8 Dans l'univers presqu'exclusivement féminin décrit dans son roman, les femmes se font porteuses de la mémoire historique du $\mathrm{xx}^{\mathrm{e}}$ siècle dans une société où les hommes sont absents, parce qu'ils ont été éliminés physiquement par la guerre, les déportations et les persécutions politiques ou parce qu'ils sont lâches et incapables d'assumer leurs responsabilités. Ce sont des femmes fortes et dignes comme les trois petites vieilles qui deviennent les artisanes du destin de Suzanna, non seulement parce qu'elles s'en occupent au quotidien et lui épargnent l'orphelinat, mais aussi et surtout parce qu'elles se vouent à la cause de préserver la fillette des mensonges de la propagande et de la mesquinerie de la morale du Parti. E. Tchijova confie aux trois grand-mères une mission de sauvegarde de la mémoire historique pour assurer que ce qui est arrivé dans le passé ne se répète plus jamais : «Les Russes doivent prendre conscience de leur histoire, sinon elle peut se répéter 6 .» 
9 Le personnage de Charlotte Lemonnier, qui se trouve au centre du roman Le Testament français de l'écrivain émigré Makine, est une autre grand-mère qui tient lieu de charnière non seulement entre le présent et le passé, mais aussi entre l'Ouest et l'Est. Les récits de cette femme extraordinaire aux yeux de tous ceux qui l'entourent et les objets mystérieux qui surgissent de sa mallette usée (des coupures de journaux, des photographies anciennes, des cartes postales jaunies par le temps et des cartes de menu) font apparaître devant le protagoniste une France grandiose, avec ses cathédrales majestueuses et ses hommes d'État éclairés, ses lumières flamboyantes et ses paysages. Le garçon grandit donc dans un environnement caractérisé par une double culture : d'un côté, les contes mythiques de sa grand-mère, qui recréent des épisodes de la vie française du début du siècle, et, de l'autre, la vie soviétique des années 1960-1970. La coexistence dans sa vie de ces deux mondes antithétiques enrichit le protagoniste en lui offrant la chance de regarder le monde d'une perspective multiple et la possibilité précieuse (et rare à l'époque) de connaître une réalité totalement différente par rapport à la société soviétique. Si, d'un coté ce dualisme permet au héros du roman de rêver de cette France féerique, il peut aussi s'avérer problématique. Comme le souligne Hélène Mélat dans sa fine analyse du récit, il comporte une crise de rejet et un procès de démystification du mythe de la France pendant l'adolescence qui ne se termine que quand, «par le biais de l'écriture, l'enfant russe est devenu écrivain français » (Mélat, 2002 : 42).

Dans ce roman, la grand-mère incarne le lien entre deux cultures mais aussi entre deux langues différentes. Le petit-fils apprend dès sa première enfance le français, un langage qu'il perçoit non pas comme étranger, mais comme un code secret qui distingue sa famille des autres, une sorte de clef magique pour accéder à un pays qui lui parait lointain et mystérieux comme une Atlantide : «[...] un pays livresque par essence, un pays composé de mots, dont les fleuves ruisselaient comme des strophes, dont les femmes pleuraient en alexandrins et les hommes s'affrontaient en sirventès. » (Makine, $1995: 292)$

\section{Le roman de formation et le rôle de la grand-mère comme référence}

« ШИРОК ЧЕЛОВЕК, СЛИШКОМ ДАЖЕ ШИРОК, Я БЫ

сузил. »

F. M. DosTOÏEVSKI

11 Dans les œuvres de formation, la grand-mère joue maintes fois un rôle éclairant. On en

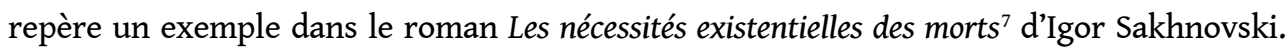
$\mathrm{Au}$ sein d'une société soviétique à la dérive qui semble n'offrir aucune chance d'élévation ou de rédemption, où dominent la désolation désespérante des villes provinciales, la mesquinerie, la corruption et l'alcoolisme, le protagoniste du roman parvient à nourrir des sentiments délicats, malgré la misère esthétique et éthique qui l'entoure, grâce à l'influence que sa grand-mère Roza, seul jalon de son enfance et adolescence, exerce sur lui.

Roza est une grand-mère " aux petits soins » : son seul but dans la vie - semble-t-il - est de veiller sur son petit-fils : «Elle veillait à mon bien-être, à l'infaillibilité de chacun de mes pas, comme si j'étais l'unique but de son existence et qu'elle n'en avait jamais eu d'autre ${ }^{8}$.» 

cette période, remplacent souvent des mères absentes, peu incisives, voire passées de vie à trépas. Sa fonction de personne de référence principale s'avère analogue à celle à laquelle fait allusion Savkina quand elle observe, à propos du personnage de la grandmère dans Le ravin de Gontcharov, dans la trilogie autobiographique de Gorki et dans Le Dernier salut d'Astafiev, que la figure de la grand-mère se présente comme un archétype, vu à travers les yeux d'un petit-fils qui est toujours un orphelin et pour lequel la grand-mère est une « mère subrogée » (Savkina, 2011). dans Sincèrement vôtre, Chourik constitue l'axe essentiel sur lequel l'existence de son petitfils repose, de sorte que, quand elle décède, la vie de Chourik semble ne plus avoir de sens, ni de direction: «Je me sens comme un train qu'on a accroché à la mauvaise locomotive et qui maintenant file à une vitesse épouvantable, sans savoir où il va ${ }^{9}$. » Chapiteau vert. Mikha tombe dans le désespoir le plus profond et perd sa capacité de réagir aux circonstances fâcheuses : "Anna Aleksandrovna était pour moi toute ma famille, je viens de le comprendre ${ }^{10}$. » Sanja n'a plus rien qui le retient d'abandonner la Russie.

Sincèrement vôtre, Chourik, L. Oulitskaïa utilise la métaphore de l'ange pour désigner le personnage de la grand-mère ( $«$ La maman et la grand-mère, deux anges aux grandes ailes $^{11} »$ ) en faisant d'elle à la fois une protectrice et une incarnation du principe féminin. Bien qu'elle meure après les premières cinquante pages du roman, sa présence continue à flotter dans le livre, à déterminer le cours des événements et à façonner le comportement de Chourik, un éternel "enfant sage" tourmenté par un sentiment de culpabilité insoutenable.

\section{Le renversement du stéréotype}

« ДЕНЬ БЫЛ НЕВИНЕН, И ВЕТЕР БЫЛ СВЕЖ. ТЕМНЫЕ ЗВЕЗДЫ ПОГАСЛИ. - БАБУШКА! - ЭТОТ ЖЕСТОКИЙ МЯТЕЖ В СЕРДЦЕ МОЕМ - НЕ ОТ ВАС ЛИ? » Marina TsVetAeVA

Si Sakhnovski confère à son héroïne un rôle déterminant - qui inclut aussi, à part le rôle plus spécifique de grand-mère, celui de mère et de femme à aimer - et idéalisé, il n'en est pas de même dans les œuvres d'autres écrivains où la description du personnage de la grand-mère, qui souvent remplace la mère, n'implique pas nécessairement un processus d'idéalisation.

Les grand-mères adoptives du Temps des femmes d'Elena Tchijova sont exemptes de toute idéalisation mais, même si elles sont très «terrestres » et pleines d'imperfections, leur fonction est salvatrice.

Mour de Ludmila Oulitskaïa, récit emblématique pour son personnage de grand-mère, présente une structure narrative tout à fait différente. L'écrivaine oppose deux modèles de grand-mères : si Anna Fedorovna, grand-mère au sens propre du terme, est une femme positive et équilibrée dans ses relations avec les enfants, on ne peut pas en dire autant de sa mère Mour. Cette dernière est une "dame de pique » dans le sens pouchkinien, une personne frivole, capricieuse et despotique avec un passé de femme fatale qui continue à 
exercer chez elle son pouvoir tyrannique sans que ni sa fille ni ses petits-enfants puissent lui tenir tête. C'est une femme-sangsue, qui a gâché la vie de sa fille, conciliante par nature, en écrasant sa personnalité et en la privant d'une propre vie en dehors du travail et de la soumission domestique. De plus, elle ressemble à un automate plutôt qu'à un être humain. L'auteur la décrit comme une machine qui avance avec un bruit de ferraille ( Faisant tinter fort les barres métalliques de son déambulateur $\left.{ }^{12} »\right)$, ou bien comme un fauve aux aguets ( Une tigresse en chasse $\left.{ }^{13} »\right)$.

Dans le monologue La Nuit m'appartient, Ludmila Petrouchevskaïa crée un personnage tourmenté de femme que les difficultés économiques, la pénurie alimentaire et les problèmes du quotidien soviétique ont endurcie jusqu'à l'exaspération, en la rendant acariâtre et malveillante à l'égard de tous, y compris ses enfants, malgré l'amour qu'elle éprouve pour eux. Anna Andrjanovna, narratrice et protagoniste de la nouvelle, est une grand-mère furibonde, en lutte contre tous et contre l'adversité. À travers son personnage, l'auteur dénonce la déshumanité de la vie soviétique qui emporte tout sans laisser d'espace pour l'élévation de l'âme. La grand-mère, bien que présentée par Petrouchevskaïa comme "poétesse ", est trop engagée dans son combat pour la survie pour pouvoir se consacrer aux raffinements de l'esprit. Il y a un seul aspect qui sape la logique intérieure d'Anna Andrjanovna en la ramenant au royaume des sentiments humains : l'amour envers son petit-fils Tima pour lequel elle est prête à tout sacrifier. Malgré le désespoir qui flotte dans ce roman, le lecteur n'arrive pas à éprouver de la compassion. Cela est dû, comme l'explique Hélène Mélat, au fait que Petrouchevskaïa introduit une sorte de décalage entre la perception de l'héroïne et celle, plus objective, du lecteur et cette « distance ironique » empêche la compassion (Mélat, 2005 : 11).

Toutefois, le véritable renversement du stéréotype de la douce mamie se produit dans le roman Enterrez-moi derrière une plinthe où Pavel Sanaev propose une image de grand-mère intentionnellement grotesque et caricaturale. L'écrivain confère à son œuvre un rythme soutenu et attribue au personnage de la grand-mère des caractéristiques monstrueuses dans sa relation avec le petit-fils à travers les yeux duquel elle nous est dévoilée. L'attention maniaque à l'égard de ce dernier, qu'elle considère comme "pourri audedans " - une véritable forme d'hypocondrie transférée sur le corps du garçon -, est associée à un caractère autoritaire et belliqueux qui trouve une issue dans les cris et les injures. La succession d'injures transforme cette nouvelle en « une fantasmagorie verbale macabre ${ }^{14} »$. Comme Juduchka (Judas) dans La Famille Golovleff de Saltykov-Chtchedrine ou Foma Fomytch de Dostoïevski dans Le Bourg de Stépantchikovo et sa population, par ses paroles - un mixte de captatio benevolentiae, d'auto-commisération et de rodomontades, de dérision et de vulgarité gratuites, de mensonge avec quelques onces de vérité - elle prend au piège l'interlocuteur, annihilant son âme et faisant de lui une victime inerte. Elle enveloppe ses victimes des nœuds de sa logorrhée en les empêchant de répondre, respirer, penser. Olga, sa fille, affirme : «Je ne sais même pas que raconter. Grand-mère a remué toutes mes pensées. Je reste là comme une poule à battre mes paupières ${ }^{15}$. »

On dirait que le renversement du stéréotype a atteint son apogée et pourtant le personnage reste, paradoxalement, un personnage de « grand-mère aux petits soins ». En dépit de l'obstination dont elle fait preuve, harcelant et hantant son petit-fils dans un crescendo où l'humour noir se mélange avec le tragique de la situation, la vieille femme est néanmoins désespérément attachée au pauvre Sacha, supposément assiégé par des staphylocoques et, entre un hurlement et une injure, lui déclare tout son amour : « Dans le monde entier il n'y a pas d'autre personne qui l'aime comme moi. Cet enfant m'a fait 
bouillir le sang. [...] je respire à travers lui, je le sens dans mes tripes ! [...] La peur pour lui c'est comme un fil qui me tire. Où qu'il est, je sens tout ${ }^{16}$. »

La présence de contradictions apparemment inconciliables rentre parmi les spécificités du genre littéraire du grotesque ${ }^{17}$. Malgré la prétendue centralité du personnage de Sacha, qui joue aussi la fonction de narrateur, la figure dominante du roman est la grandmère, et c'est par le code interprétatif de cette dernière que l'enfant perçoit la réalité soviétique de ces années. La vision de la grand-mère fait que les événements acquièrent parfois une tonalité absurde et que le monde extérieur apparaît comme le prolongement d'un contexte familial asphyxiant, une réalité claustrophobique où la seule opportunité d'évasion est offerte par les rêves.

En revanche, dans les œuvres mentionnées de L. Oulitskaïa, E. Tchijova, A. Makine et I. Sakhnovski, le personnage de la grand-mère représente un élément de continuité, un trait d'union implicite avec la culture, l'histoire et parfois la langue d'une autre époque ou d'un autre lieu, qui permet d'outrepasser la réalité soviétique.

\section{BIBLIOGRAPHIE}

AGEEV Aleksandr (2006), « O pol'ze babušek », Znamja, 5.

AstAF'EV Viktor (1968), Poslednij poklon [Le dernier salut], Perm : Permskoe knižnoe izd.

ČIžova Elena (2010), « Ja pisala roman dlja teh, kto umer », Novaja gazeta, 12 mars.

Čižova Elena (2012), Vremja ženščin, Moscou : Ast.

DENEŽKINA Irina (2006), « Babulja », Ural, 3.

GONČAROV Ivan (1869), « Obryv » [Le ravin], Vestnik Evropy, 1-5.

GORKI Maxime (2005), « Trilogie : Enfance, En gagnant mon pain, Mes universités », Euvres, Paris :

Gallimard, coll. « La Pléïade » [publication originale en russe : 1913-1914].

MAKINE André (1995), Le Testament français, Paris : Mercure de France.

MÉLAT Hélène (2002), « Andreï Makine : Testament français ou Testament russe ? », La Revue russe, 21, 41-49.

MÉLAT Hélène (2005), « Du traumatisme à l'évasion : les premiers pas de la littérature postsoviétique (1992-2005) », La Revue russe, 26, 9-24.

OULITSKAÏA Ludmila (1998), Médée et ses enfants, trad. Sophie Benech, Paris : Gallimard.

OULITSKAÏA Ludmila (2005), Sincèrement, vôtre, Chourik, trad. Sophie Benech, Paris : Gallimard.

OULITSKAÏA Ludmila (2014), Le chapiteau vert, trad. Sophie Benech, Paris : Gallimard.

SAHNOVSKIJ Igor' (1999), « Nasuščnye nuždy umerščix », Ščastlivcy i bezumcy, Moscou : Vagrius.

SAKHNOVSKI Igor (2007), Roza, Paris : Gallimard.

SANAEV Pavel (2014), Pohoronite menja za plintusom, Moscou : Ast. 
SANAIEv Pavel (2010), Enterrez-moi sous le carrelage, trad. Bernard Kreise, Paris : 10/18 Poche.

SAVKINA Irina (2011), « U nas nikogda uže ne budet etih babušek? », Voprosy literatury, 2.

Tchijova Elena (2009), Le temps des femmes, trad. Marianne Gourg-Antuszewicz, Lausanne : Noir sur blanc.

UlicKAJA Ljudmila (1996), Medeja i ee deti, Moscou : Vagrius.

UlıCKAJA Ljudmila (2004), Iskrenne vaš Šurik, Moscou : Eksmo.

Ulickaja Ljudmila (2011a), « Mur », Pervye i poslednie, Moscou : Astrel'.

UliCKAJA Ljudmila (2011b), Zelenyj Šater, Moscou : Eksmo.

\section{NOTES}

1. La composition traditionnelle de la famille change suite aux événements qui se passent dans la société soviétique - et après dans la société russe - en raison de facteurs comme l'alcoolisme, le nombre croissant de ménages dans lesquels il n'y a presque que des femmes : la grand-mère, la mère et les enfants et aussi de l'augmentation des familles recomposées.

2. «А ЧЕРТ ЕГО ЗНАЕТ, МОЖЕТ, И ВПРЯМЬ НА БАБУШКАХ ДЕРЖИТСЯ РУССКАЯ КУЛЬТУРА?», (Ageev, 2006)

3. « С ГОДАМИ ЕЕ ПРЕКРАСНЫЕ ИСТОРИИ ЗАСТЫЛИ, ОТВЕРДЕЛИ И, КАЗАЛОСЬ, НЕВИДИМЫМИ КЛУБКАМИ ЛЕЖАЛИ В ЯЩИКЕ ЕЕ СЕКРЕТЕРА РЯДОМ С ФОТОГРАФИЯМИ. » (Ulickaja, 2004 : 21)

4. « БАБУШКА ‘ПИТАЕТ’ ВНУКА В ПРЯМОМ И ПЕРЕНОСНОМ СМЫСЛАХ » [La grand-mère «nourrit » son petit-fils dans un sens littéral et figuré] (Savkina, 2011).

5. L. oulitskaïa, Médée et ses enfants, Paris : Gallimard, p. 197. Texte russe : « это БЫл НАСтоящиЙ СЕМЕЙНЫЙ АРХИВ, И, КАК ВСЯКИЙ НАСТОЯЩИЙ АРХИВ, ОН УКРЫВАЛ НЕРАЗГЛАСИМЫЕ ТАЙНЫ.» ( Ulickaja, $1996: 165)$

6. « РУССКИМ НЕОБХОДИМО ОСМЫСЛИТЬ СВОЮ ИСТОРИЮ, ИНАЧЕ ОНА МОЖЕТ ПОВТОРИТЬСЯ.» (С̌ižova, 2010)

7. Paru en France sous le titre de Roza, Paris : Gallimard, 2007.

8. « ОНА СПОКОЙНО И ТЩАТЕЛЬНО СЛЕДИТ ЗА МОИМ БЛАГОПОЛУЧИЕМ, ЗА БЕЗОШИБОЧНОСТЬЮ КАЖДОГО МОЕГО ШАГА - И КАЖЕТСЯ, ЧТО НИКАКИХ ДРУГИХ ЦЕЛЕЙ В ЕЁ ЖИЗНИ НЕ БЫЛО И НЕТ.» (Sakhnovski, 2007 : 15) Texte russe : Sahnovskij (1999: 168).

9. « Я ЧУВСТВУЮ СЕБЯ ПОЕЗДОМ, КОТОРЫЙ ПРИЦЕПИЛИ К ЧУЖОМУ ПАРОВОЗУ И ОН ЛЕТИТ СО СТРАШНОЙ СКОРОСТЬЮ, НО НЕ ЗНАЕТ САМ, КУДА. » (Ulickaja, 2004 : 171)

10. « АННА АЛЕКСАНДРОВНА БЫЛА МНЕ ВСЕЙ РОДНЕЙ, ВМЕСТЕ ВЗЯТОЙ, Я ТОЛЬКО СЕЙЧАС ЭТО ПОНЯЛ. ( Ulickaja, 2011b : 500)

11. « МАМА И БАБУШКА, ДВА ШИРОКОКРЫЛЫХ АНГЕЛА » (Ulickaja, 2004 : 31).

12. « ТОНКО ПОЗВЯКИВАЯ МЕТАЛЛИЧЕСКИМИ ПЛАНКАМИ ХОДУНКОВ » (Ulickaja, 2011a : 213).

13. « ТИГРИЦА НА ОХОТЕ » (Ulickaja, 2011a : 228).

14. Selon la définition très appropriée d'Anastassia Vinogradova-de La Fortelle dans l'article «Enterrez-moi derrière une plinthe : une écriture de la peur ou une écriture cathartique?", Chroniques slaves, $\mathrm{n}^{\circ}$ 2, 2006, p. 165.

15. «НЕ ЗНАЮ ДАЖЕ, ЧТО И РАССКАЗАТЬ. БАБУШКА МНЕ ВСЕ МЫСЛИ СМЕШАЛА, СИЖУ, КАК КУРИЦА, ГЛАЗАМИ ХЛОПАЮ. » (Sanaev, 2014 : 242)

16. « А ТАК - НЕТ НА СВЕТЕ ЧЕЛОВЕКА, КОТОРЫЙ ЛЮБИТ ЕГО, КАК Я. КРОВЬЮ ПРИКИПЕЛО КО МНЕ ДИТЯ ЭТО. [...] Я ДЫШУ ИМ, ЧУВСТВАМИ ЕГО ЧУВСТВУЮ! [...] СТРАХ ЗА НЕГО, КАК НИТЬ, ТЯНЕТСЯ, ГДЕ БЫ НИ БЫЛ, ВСЕ чУВСТВУю. » (Sanaev, 2014 : 184-185) 
17. «Les contradictions et les antinomies sont vraiment centrales dans l'art et la littérature grotesques. » (A. castore, Grottesco e riscrittura, Rome : Aracne, 2012, p. 15)

\section{RÉSUMÉS}

Parmi les nombreux personnages qui peuplent la littérature russe contemporaine, l'image de la babouchka se distingue par son importance. Cela est dû non seulement à la personnalité des grand-mères et aux valeurs qu'elles relayent, mais aussi au rôle qu'elles ont joué, et parfois jouent encore, dans la société russe en vertu de circonstances historiques particulières et de l'organisation de la vie sociale, surtout à l'époque soviétique.

Un examen des œuvres de quelques écrivains de la période de la seconde moitié du $\mathrm{xx}^{\mathrm{e}}$ siècle à nos jours (L. Oulitskaïa, E. Tchijova, I. Sakhnovski, L. Petrouchevskaïa) révèle une série de personnages de babouchka qui représentent un élément fondamental de continuité sur le plan historique et culturel au sens large et qui proposent, par leurs gestes et leurs paroles, un patrimoine d'humanité, d'idéaux et de simple gestion du quotidien à l'opposé de la doctrine officielle et une autre façon de regarder et interpréter la réalité. Les grand-mères, en outre, jouent souvent un rôle déterminant - qui inclut, à part celui plus spécifique de grand-mère, aussi le rôle de mère et de femme à aimer - et ont parfois une fonction salvatrice. La représentation littéraire de la grand-mère se déroule aussi bien dans le respect des canons de la tradition, que par le biais du renversement du stéréotype de la douce mamie.

ОСОБОЕ МЕСТО, КОТОРОЕ ЗАНИМАЕТ ОБРАЗ БАБУШКИ В СОВРЕМЕННОЙ РУССКОЙ ЛИТЕРАТУРЕ НЕ СООТВЕТСТВУЕТ ТОЛЬКО РОЛИ, КОТОРУЮ БАБУШКА ИСПОЛНЯЛА И ПРОДОЛЖАЕТ ИСПОЛНЯТЬ В СИЛУ СЛОЖИВШИХСЯ В СОВЕТСКУЮ ЭПОХУ ОСОБЕННОСТЕЙ ОРГАНИЗАЦИИ СОЦИАЛЬНОЙ ЖИЗНИ, НО ОТРАЖАЕТ ТАКЖЕ НЕКОТОРЫЕ ВАЖНЫЕ ЛИТЕРАТУРНЫЕ ФУНКЦИИ, КОТОРЫЕ С ЭТИМ ОБРАЗОМ АССОЦИИРУЮТСЯ. БАБУШКИ ЧАСТО ЯВЛЯЮТСЯ СВЯЗУЮЩИМ ЗВЕНОМ МЕЖДУ РАЗЛИЧНЫМИ ЭПОХАМИ, ЗВЕНОМ В НЕПРЕРЫВНОМ ПРОСТРАНСТВЕННО-ВРЕМЕННОМ КОНТИНУУМЕ, ИНОГДА ЗАМЕНЯЮТ МАТЕРЕЙ И ДАЖЕ ЛЮБИМЫХ ЖЕНЩИН. В НЕКОТОРЫХ СЛУЧАЯХ ЛИТЕРАТУРНОЕ ИЗОБРАЖЕНИЕ ОТСТУПАЕТ ОТ ТРАДИЦИОННЫХ КАНОНОВ И ПРИБЕГАЕТ К СВЕРЖЕНИЮ СТЕРЕОТИПА ДОБРОЙ БАБУЛИ.

\section{INDEX}

Mots-clés : grand-mère, littérature russe contemporaine, mémoire historique du XXe siècle, babouchka, charnière entre le présent et le passé, Ludmila Oulitskaïa motsclesru БАБУШКА, СОВРЕМЕННАЯ РУССКАЯ ЛИТЕРАТУРА, ИСТОРИЧЕСКАЯ ПАМЯТЬ ХХ ВЕКА, СВЯЗУЮЩЕЕ ЗВЕНО, ЛИТЕРАТУРНЫЙ ОБРАЗ БАБУШКИ, ЛЮДМИЛА УЛИЦКАЯ

\section{AUTEUR}

\section{GIULIA GIGANTE}

Université libre de Bruxelles (Belgique) 